there were some boys throwing stones at one another in play. One of the stones hit the left lens punching a hole in the middle and producing this interesting radiate fracture. It is a $-7 \mathrm{D}$. sph. It will be noticed that at its centre it is hardly $0.5 \mathrm{~mm}$. in thickness.

The impact could not have been very strong, as is evidenced by the retention of the fractured fragments in position in the frame and by the absence of any damage to the eye of the boy.

The next case is one of a man who, while watching a boat under repair at the Southampton Docks, had a bolt fly from the boat and strike his spectacles. The left lens was shivered to fine bits. He came in a hurry to the hospital fearing that something serious had happened to his left eye. All the injury he had sustained was a slight peripheral abrasion of the cornea. At the outer canthus and among the lashes there were a few fine flakes of glass which could easily pass for bits of broken cover slips such as are used in bacteriological microscopy.

The patient was a myope wearing $-4 \mathrm{D}$. spheres.

\title{
A NEW HETEROPHORIA TEST
}

\author{
BY \\ A. R. Brailey, \\ LONDON.
}

THIS simple apparatus was devised for the detection and estimation of heterophoria in candidates for the Royal Air Force. In use it has proved so rapid and efficacious that I think it may well supplant the Maddox rod in ordinary practice. In principle it depends on the old red and green letter test for binocular vision. Illuminated green letters are seen by one eye through a green glass, a red spot is seen by the other eye through a red glass. The letters are disposed in two rows, one above the other, and are placed so that the vertical limb of each letter in either row is at a distance, the one from the other, of the tangent of two degrees at six metres. Circular spots on the vertical limbs of each letter are at a distance of the tangent of half a degree from each other; on the horizontal limbs the distance of the tangent is one degree. The red spot, by a simple shutter, can be shifted to either of two positions. The position of the red spot as described by the patient in relation to the letters, at once denotes the presence, variety, and degree of heterophoria.

The advantages over the Maddox rod are:

1. That the amount of heterophoria is determined at once.

2. That by means of a rotating prism it is easy in a moment to see what correction is necessary to restore perfect muscle balance. 
3. That by shifting the position of the red spot the range of the scale is increased, and it is impossible for the patient to know what its position should be in relation to the letters.

4. That patients with any degree of binocular vision at all apparently find no difficulty in seeing the spot and the letters. With the Maddox rod, it is often very difficult to get them to see both the light and the streak.

It is not necessary that the test should be carried out in a dark room, but the light must not be sufficient for the patient to see more than the red spot with the one eye, or the results are vitiated by the fusion sense. The apparatus can be obtained from Messrs. C. W. Dixey \& Son, 3, New Bond Street, London.

\section{A CASE OF AN EYELASH PERFORATING THE CORNEA AND ANTERIOR LENS CAPSULE}

BY

R. Graham Brown, BRISBANE.

No. 7363 Pte. A. C-—, 1st Wiltshire Reg., aged 26 years. This patient was seen by me on September 26, 1914, at No. 3 General Hospital at St. Nazaire. He had been shot through the left lower jaw on the 20th September. (My notes do not give more details on this point. I am, however, almost sure that the wound entrance was inside the mandible below, and the exit on the cheek just below the left lower lid). The patient stated that he suffered no pain in the eye until about two minutes after the wound when "the eye felt as if it was burning all over." $\mathrm{He}$ was told that it was blistered. The same night he had swelling of the eyelids. Until seen by me (six days after he was wounded) no treatment of the eye condition had been carried out. I found that there was a great deal of chemosis, which almost covered the whole of the cornea. There was no purulent discharge. The tension was not increased, and there was no ciliary tenderness. An anaesthetic was given to allow of a complete examination of the cornea. A deeply infiltrated area of about $2 \mathrm{~mm}$. in diameter was discovered at about 6 o'clock, and 1 to $1 \frac{1}{2} \mathrm{~mm}$. from the limbus. On gently probing the infiltrated area, which was, by the way, sodden on the surface, a small black foreign body was seen. On seizing this with a pair of iris forceps and applying traction, an eyelash was withdrawn. This surprised us not a little. My notes say, "Eyelash $4 \mathrm{~mm}$. long-? from the lower lid." The iris was clear and not contracted, and the lash had penetrated the lens capsule. Atropin was instilled; and fomentations were applied to the orbital region. 